\title{
State Machine Antipatterns for UML-RT
}

\author{
Tuhin Kanti Das \\ School of Computing \\ Queen's University \\ Kingston, Canada \\ Email: das@cs.queensu.ca
}

\author{
Juergen Dingel \\ School of Computing \\ Queen's University \\ Kingston, Canada \\ Email: dingel@cs.queensu.ca
}

\begin{abstract}
Software development guidelines are a set of rules which can help improve the quality of software. These rules are defined on the basis of experience gained by the software development community over time. Software antipatterns are a powerful and effective form of guidelines used for the identification of bad design choices and development practices that often lead to poor-quality software. This paper introduces a set of seven state machine antipatterns for the model-based development of real time embedded software systems. Each of these antipatterns is described with a pair of examples: one for the antipattern itself and a second one for improved, refactored solution.
\end{abstract}

\section{INTRODUCTION}

Complex distributed real-time software systems are most frequently encountered in telecommunications, aerospace, defense and automatic control applications. Although modern programming languages as well as the supporting integrated development environments (IDEs) have advanced significantly, developing these complex embedded software systems using current code-centric technologies still requires grueling effort.

The vision of Model Driven Engineering (MDE) has been introduced to mitigate this problem by allowing for systems to be described at multiple levels of abstraction and providing automated support for transforming and analyzing models [1]. As an example, for the analysis and design of complex embedded software in the telecommunications industry, an architectural description language named Real-time ObjectOriented Modeling (ROOM) has been introduced in [2]. ROOM was aligned with the Unified Modeling Language (UML) which gave rise to the real-time profile of UML (UML-RT). UML-RT supports constructs for modeling the structural and behavioral properties of a real-time system. These modeling constructs have been represented using the industry standard UML for attaining the benefits of popular and widely-supported notations. The concepts of ROOM are incorporated into the Model Driven Engineering (MDE) tools IBM Rational Rose Real-Time (RoseRT), IBM RSA-RTE and the open source tool Papyrus-RT in the form of UML-RT [3].

A software design model is an abstract representation of a system. It is the primary artifact in the MDE vision of software development, and computer-based technologies are used to transform the design models to the running systems. Designing a system involves the process of deriving a design model from requirement specifications from which a system can be generated more or less automatically [4]. The MDE tools mentioned above use UML-RT for compiling design models into code and link it with the runtime system.
Due to the high initial cost for developing real time software systems, preference is given to modifying the software instead of rewriting it when major new requirements are identified. Therefore, a well-designed architecture is extremely important for developing real-time software systems which in addition to simplifying the initial construction, promotes the evolution of the system [3].

A software antipattern is used for describing a commonly occurring solution to a problem having some decidedly negative consequences. Antipatterns serve two purposes at the same time: they help developers identify bad design practices and they assist in refactoring the problematic solutions. Therefore, antipatterns play an important role in improving the quality of real-time software systems by assisting developers to avoid bad designs. In this paper, we introduce a set of seven behavioral antipatterns for the development of model-based real-time embedded software systems. These antipatterns are the result of our study of almost 100 UML-RT models created in industry and academia. A standard format for describing antipattern introduced in [5] is followed for presenting these antipatterns, and the examples for demonstrating the antipatterns are created using the IBM RSA-RTE tool.

This paper is structured in the following way: Section 2 presents relevant background terminology for software development guidelines in general. This is followed by a brief summary of existing work on antipatterns. Finally, the antipattern set is introduced in Section 4.

\section{BACKGROUND}

\section{A. Software Quality}

In the area of Software Engineering, two related but distinct notions exist whenever software quality is defined in a business context: Software functional quality and software structural quality. The conformance of software to a given design, based on functional requirements or specifications is represented by software functional quality [6]. It is typically enforced and measured through software testing. On the other hand, software structural quality reflects the degree to which software is produced correctly. So, it represents how well software complies with the non-functional requirements such as reliability or maintainability that support the delivery of the functional requirements. Effectively, this is how the architecture of software adheres to the sound principles of software architecture outlined in [7].

For a piece of software to provide business value, the Consortium for IT Software Quality (CISQ) has defined five 
major desirable structural characteristics on the basis of ISO 9126-3 and the subsequent ISO 25000:2005 [8] quality model: Reliability, efficiency, security, maintainability and (adequate) size.

Measuring software quality is mainly motivated by two facts: risk management and cost management. In addition to causing inconveniences, historically software failures have caused human fatalities. An example of a programming error that led to multiple deaths is discussed in [9]. This enforces the requirement of regulating the development of certain types of software, particularly software embedded in medical and other devices. In addition, an application with good structural software quality is much easier to understand and change in response to evolving business needs. Therefore, the associated cost for maintaining such software is significantly less.

\section{B. Definitions and Terminology}

In general, every software development team maintains a well-defined set of software development guidelines for achieving software quality attributes. These guidelines are defined as a set of recommended best practices that must be followed to improve the productivity of development teams and to produce consistent deliverables [10]. In addition to software development conventions, which are standards recommended to be followed strictly in a development community, software development guidelines can also include a set of software development principles which are usually defined with some tradeoffs. A set of principles may be useful in some situations, but not in others. A discussion on software development principles includes a set of patterns, antipatterns and smells [10]-[14].

A software design pattern is a general reusable solution to a commonly occurring problem within a given context in software design. Thus the core sections that constitute a design pattern are the description of a problem followed by a solution to the problem. On the contrary, an antipattern consists of two solutions: a problematic solution followed by a refactored solution. The concepts of patterns and antipatterns are related to each other. The main difference is the application context. A pattern solution can be treated as a problematic solution in an antipattern if it is applied in an inappropriate context.

While the discussion of patterns and antipatterns involve identification and refactoring of a problem, smells are considered as indications of weaknesses in code or design that may slow down development or increase the risk of bugs or failures in future [11]. Martin Fowler defined a smell as a surface indication that usually corresponds to a deeper problem in the system [15].

Compared to the templates available in the literature for discussing patterns [16], [17], antipattern templates [5] have been found to be much more powerful and effective for describing recurring solutions. The advantages of using the latter include the amplification of the problem in a way that can help readers recognize the problematic structure, symptoms and consequences. Moreover, it also gives the flexibility to include the discussion of any alternative known solutions for improving the problematic one, in addition to the refactored solution. These advantages motivate us to write up our findings following the antipattern template introduced in [5].

\section{RELATED WORK}

Since the introduction of the term design pattern in 1994, there has been an exceptional growth in the publication of design pattern literature. On the bright side, a large and growing base of reusable design solutions that can be evaluated and applied to a software development effort is available for skilled object oriented developers. However, the potential pitfalls of using design patterns became visible when many developers failed to properly evaluate the applicability of a particular design pattern in their problem context [5]. To address the challenge of using design pattern, Michael Akroyd first came up with a formal model for antipatterns based on a detailed analysis of the object-oriented literature [18].

\section{A. Antipatterns in Code}

Most of the works done on antipatterns are related to codedriven development. For investigating whether the presence of two antipatterns, called Blob and Spaghetti Code, affect developers' understandability of systems during comprehension and maintenance task, an empirical study is conducted in [19]. According to their investigation, the combination of these two antipatterns can make it difficult to understand the affected system. In [20], antipatterns are represented as a set of rules. A methodology is introduced for detecting Enterprise JavaBeans (EJB) antipatterns which focuses on comparing the pre-defined rules with the application properties. Some other research work regarding antipatterns in code includes an investigation of the impact of antipatterns on change and fault-proneness of the classes of object-oriented systems [21], [22] and the use of antipatterns to predict bugs [23].

\section{B. Antipatterns in Models}

A performance antipattern identifies a practice that badly affects performance, and it may involve static and dynamic aspects of software as well as deployment features. A number of work is done considering the detection and refactoring of performance antipatterns. The authors in [24] specify a set of 14 performance antipatterns on the basis of their practical experience. Based on this research work several techniques have been introduced aimed at detecting performance antipatterns in software architectural models [25], [26]. The authors in [25] show how performance antipatterns can be defined and detected in UML models using OCL. They show with an example that the removal of a certain antipattern actually allows overcoming a specific performance problem by presenting a case study in UML annotated with the MARTE profile. For identifying performance antipatterns in Palladio architectural models and removing them, an approach is presented on the basis of rules and actions in [26]. This approach formalizes the informal existing definitions of performance antipatterns. In [27], the authors undertake the problem of removing performance antipatterns detected in an architectural model. They use a Role-Based Modeling Language to represent antipattern problems and solutions as Source Role Models (SRMs) and Target Role Models (TRMs) respectively. In their procedure, model refactoring for removing antipattern is done by replacing an SRM with the corresponding TRM. However, we have not found any existing work on antipatterns considering behavioral diagrams of UML-RT. 
Proper documentation of an antipattern describes a general form; the primary causes which led to the general form; symptoms that describe the way of recognizing the general form; the consequences of the general form; and a refactored solution demonstrating how the code or the model can be changed to resolve the problem expressed by the antipattern. In [5], the authors present a catalog of 40 antipatterns from three major viewpoints: the software developer, the software architect, and the software manager. They introduce a number of templates for antipattern description. The choice of a specific template for a new antipattern depends on the needs of the audience and the resources available to the author of the antipattern. The use of these templates ensures the inclusion of answers to important questions while describing an antipattern. For example, the full antipattern template introduced in [5] gives the flexibility to antipattern authors to include a number of optional sections in addition to two core sections: general form of the antipattern and the refactored solution. It allows the background discussion of an antipattern to be written up under a separate heading. Antipatterns are not always wrong and there are certain scenarios when using antipattern solutions is not a bad idea. The full template allows the antipattern writers to mention these exceptional scenarios under the heading of "Known Exceptions". In addition, if there are known alternative solutions available to the antipattern apart from the refactored solution, they can be discussed as "Variations".

\section{The Antipattern SET}

This section introduces a set of seven antipatterns in the behavioral design of UML-RT. These antipatterns are outcomes of our analysis of almost 100 UML-RT models in industry and academia. In addition to investigating a repository of anonymized student models considering the design of a simple Electronic Warfare System (EWS) in Royal Military College of Canada, inspecting a number of telecommunication models at Ericsson Inc. during a research visit of six weeks helped us identifying these antipatterns.

The first three entries in this set introduce antipatterns in UML-RT's decision-making processes and demonstrate corresponding refactored solutions. There exist two types of decision-making processes in UML-RT state machines: static conditional branches and dynamic conditional branches. They differ with respect to when a guard is evaluated. The purpose of these branches is to direct the behavior of a state machine at the branching locations according to the specification. This is followed by the introduction of two antipatterns for transitions in UML-RT state machines. There are three types of transitions available in state machine diagrams: external, internal and local. The difference among these transition types lies in the execution behavior for the entry and exit code of the corresponding state. Finally, we introduce two other antipatterns which consider different types of signal communication and the inheritance feature in UML-RT respectively. The examples we demonstrated for the antipatterns $D, F$ and $G$ in this antipattern set are inspired by the student model repository we used for analyzing UML-RT models.

\section{A. Incorrect use of Junction Points}

\section{Background}

In state machine diagrams, dynamic conditional branches are represented by choice-points. A choice-point is a vertex that can have one or more incoming transitions as well as one or more outgoing transitions. The decision of following a particular outgoing transition is made dynamically, after taking the incoming transition. On the other hand, junctionpoints represent static conditional branches in state machine diagrams. Similar to a choice-point construct, a junction-point is a vertex that allows one or more incoming transitions and one or more outgoing transitions. However, in contrast to a choice-point, the decision of taking a particular outgoing branch from a junction-point is made statically, before taking the incoming transition. So, the difference between static and dynamic branches in state machine diagrams is whether the guards of outgoing transitions are evaluated before (static) or after (dynamic) the incoming transition is taken. Static conditional branches allow a transition path to be determined before the execution of the incoming transition effect, whereas their dynamic counterparts do not take a transition path until the incoming transition effect has been executed [28].

For example, in Figure 11. static conditional branching is used. When the trigger for $t l$ transition is fired, the guard conditions of $t 1$ and $t 2$, if there are any, will be evaluated before executing any action code in $t 1$ and $t 2$. Now, since $X=1$ and $\mathrm{Y}=1$ in the Begin state and no guard condition is specified for $t 1$ transition, only the guard condition for transition $t 2$ will be checked which evaluates to true. So, the action code of $t 1$ and $t 2$ will be executed and the execution will move to the End state.

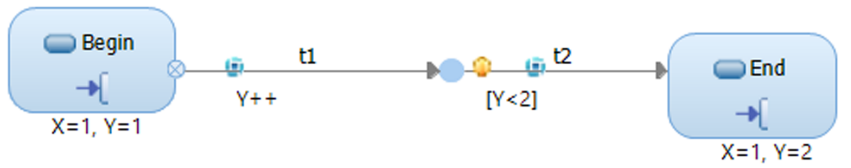

Fig. 1: Static Conditional Branching (Junction-point)

In contrast, in Figure 2, as dynamic conditional branching is used, when the trigger for $t l$ transition is fired, since no guard condition is defined for $t l$ transition, the action code of $t 1$ will be executed and the execution will come to the choice-point $C P$. Now, with $\mathrm{Y}=2$, the guard condition for $t 2$ will evaluate to false, and the execution will take the Else transition for going to Default state.

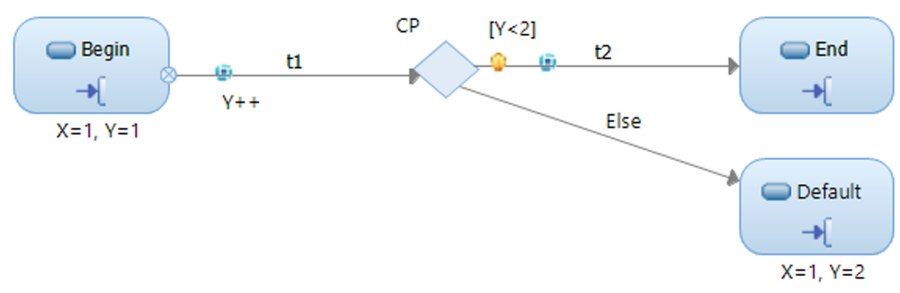

Fig. 2: Dynamic Conditional Branching (Choice-point)

\section{General Form}

Let us consider the scenario of a vending machine in Figure 3. In this example, initially the value of the variable drinkType is assigned "none" in StateA. Once the user requests a drink, the input transition is triggered where the drinkType variable is supposed to be updated with the requested drink-type "X". 


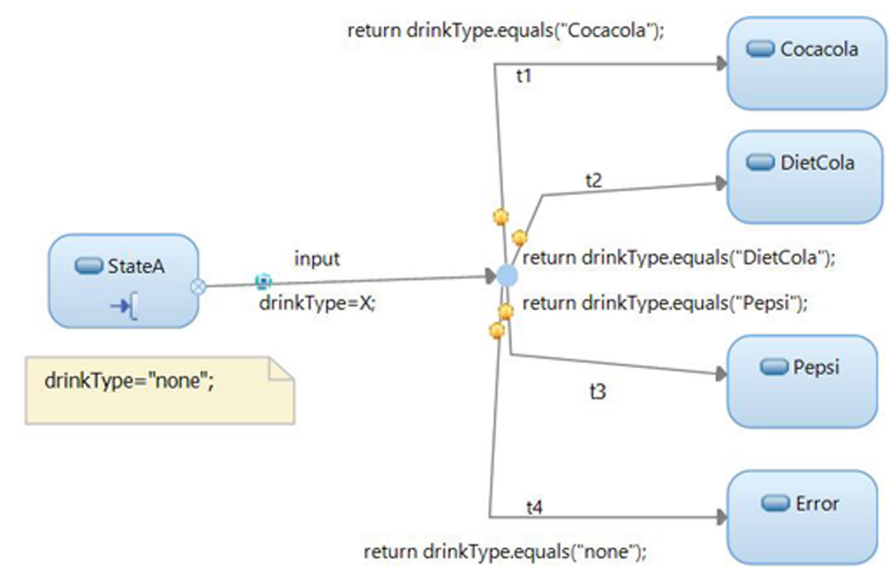

Fig. 3: Incorrect use of junction points: the drinkType variable is updated in the incoming transition (input) and is affecting the guard conditions of the outgoing transitions

Now, as a junction-point is used here which represents static conditional branches, the choice will be made solely by evaluating the guard conditions associated with the transitions before executing the action code of input transition. So, the value of the input will be ignored and the machine will always transition to the Error state.

\section{Symptoms and Consequences}

- Due to the subtle, potentially confusing semantic difference between choice-points and junction-points, their use can have some adverse effects on understandability and may lead to incorrect models, especially for novice UML-RT users.

\section{Refactored Solution}

This antipattern solution can be resolved by replacing the static conditional branching with dynamic conditional branching. If we incorporate a choice-point in place of the junctionpoint used in the antipattern solution, the action code of input transition will get executed before evaluating the guards of outgoing transitions, and consequently, the execution would take the expected path depending on the user input. Thus, the refactored solution would eliminate the possibility of any potential puzzlement in the design model.

\section{Known Exceptions}

If the action code of the transition to the junction point does not affect its guard, we can use junction-points. Otherwise, choice-points should be used.

\section{B. Overlapping Conditionals}

\section{Background}

Choice-points allow for dynamic conditional branches. The purpose of using a choice-point in state machine is similar to that of using an if-else block or a switch statement in code. It is important to use the guard conditions associated with the outgoing transitions of a choice-point properly.

\section{General Form}

Let us consider the scenario in Figure 4(a) which shows an if-else block used in classical code-oriented programming with overlapping conditions in code. Although it is not considered good practice to implement an if-else block in this way, the execution behavior in this code segment is clearly defined. The conditions in the if-else block will be checked in the sequence of their order of appearance in the code and consequently, the code block associated with the textually first condition evaluating to true will be executed.

Now, let us consider a similar scenario in the context of model-driven development in Figure 4(b). What is the execution behavior of this model going to be?

According to our observations in the RSA-RTE tool, in $C P 1$, the outgoing transition that is created first among the set of the outgoing transitions having true guard conditions will get executed. Consequently, without knowing in which order the branches have been created, it is impossible to predict the execution behavior of this model.

\section{Symptoms and Consequences}

- Overlapping conditions in choice-points affect the understandability and maintainability of the model.

- This antipattern solution can lead the system to an erroneous state. For example, in Figure 4(b), let us assume that the order of creation of the outgoing transitions in $\mathrm{CP} 1$ is as follows: $t 3, t 4, t 1, t 2, t 5$, False. Since the transition $t 3$ is created first among the outgoing transitions having true guard conditions, the execution will follow the $t 3$ transition for going to the State_X_GT_l state. Now, if the $t 3$ transition is removed from the model accidentally and reintroduced again, surprisingly, the execution behavior will not remain the same. In this case, instead of taking the $t 3$ transition, the execution will take the $t 4$ transition for going to the State_X_GT_3 state. This unexpected change in the model behavior can easily lead the system to an erroneous state.

\section{Refactored Solution}

This antipattern solution can be resolved by introducing a chain of choice-points. As we can see in Figure 5, the difficulty in understanding the execution behavior of the antipattern solution is eliminated in the refactored solution. As a result, after having a look at the model it can be easily concluded that the execution will take the outgoing transition $t 3$ of $C P \_l$ as it appears first in the chain of choice-points. The refactored solution protects the design from exhibiting unexpected behavior, which improves the consistency, understandability and maintainability of the model significantly. The use of extra choice-points reduces the performance of the model. However, considering the improvement in the quality attributes mentioned above, the cost in performance is acceptable.

\section{Known Exceptions}

If no overlapping conditions exist in the guards of outgoing branches from a choice-point, no refactoring is required as the model with single UML-RT choice-point executes faster since it contains fewer model elements.

\section{Choice-point with non-exhaustive guards}

\section{General Form}




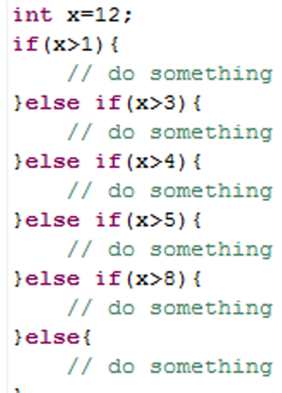

(a)

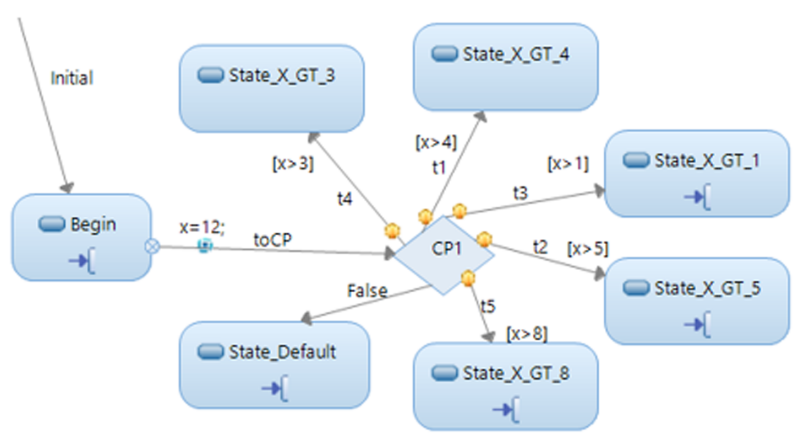

(b)

Fig. 4: Overlapping conditions in guards of code-driven and model-driven development

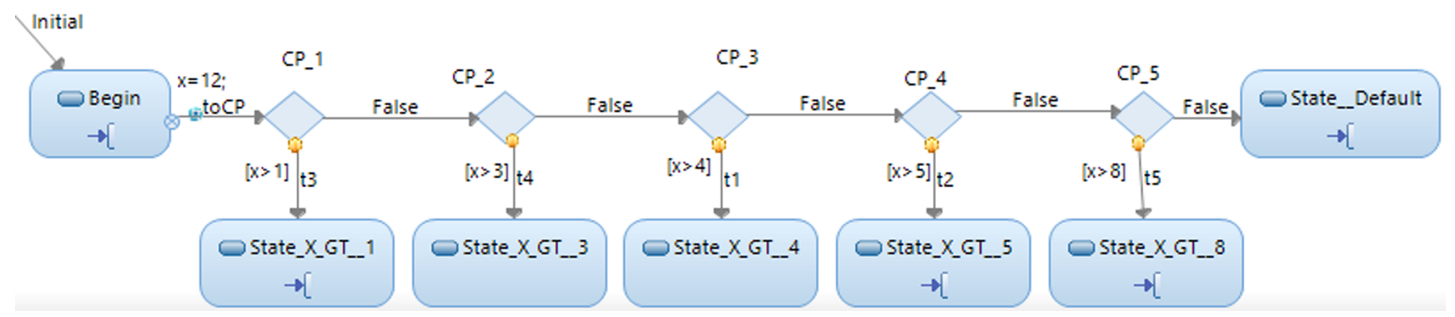

Fig. 5: Breaking overlapping conditions

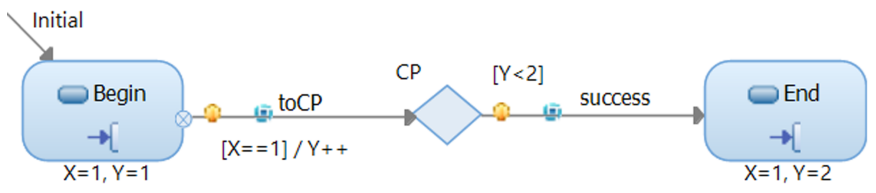

Fig. 6: An ill-formed choice-point

Let us consider the example shown in Figure 6. As we can see, there is no Else guard associated with the choice-point $C P$. Now, the question is what will happen if the event trigger for toCP transition arrives while the execution is in the Begin state?

As the choice-point model element represents dynamic conditional branch, after evaluating the associated guard condition for $t o C P$ transition to true, the corresponding action code of toCP transition will get executed and the execution will arrive in the choice-point $C P$. So, before arriving in $C P$, the value of $Y$ will be updated and consequently, it makes the guard condition of the outgoing transition success false. This causes the execution to get stuck in the $C P$ element. Now, unlike a state model construct, a choice-point element cannot handle any signal events.

It is to be noted here that, if a junction-point is used in a similar situation like this, due to its static conditional characteristic, deadlock cannot occur. If no valid path is found after evaluating all the available outgoing transitions from a junction-point, the execution will go back to the state it was in earlier.

\section{Symptoms and Consequences}

- Absence of Else guard in a choice-point.

- This antipattern could cause deadlock.

\section{Refactored Solution}

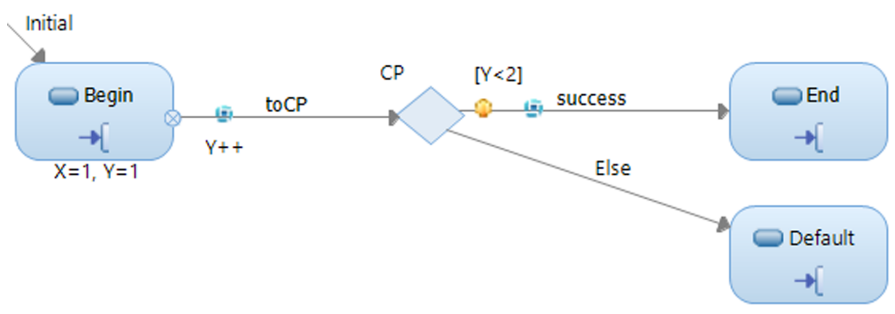

Fig. 7: A well-formed choice-point

The problem created by this antipattern can be avoided by introducing an Else guard with every choice-point construct used in the model design. As we can see in Figure 7, due to the presence of the Else guard, the possibility of having a deadlock situation is eliminated and the execution will move to the Default state.

\section{Inappropriate use of self-transitions}

\section{Background}

Self-transitions are available in all three forms of transitions in UML-RT state machines. The difference between different types of self-transition is in the execution behavior of the entry and exit code for the enclosing state.

\section{General Form}

A key benefit of MDE is the ease of understanding a particular design model through the visualization of interactions among modeling constructs. If the signals can only be received in a certain order, then this should be reflected in the state machine. It makes the state machine easier to understand, it likely improves the correlation with product documentation and errors are likely to be detected earlier. 


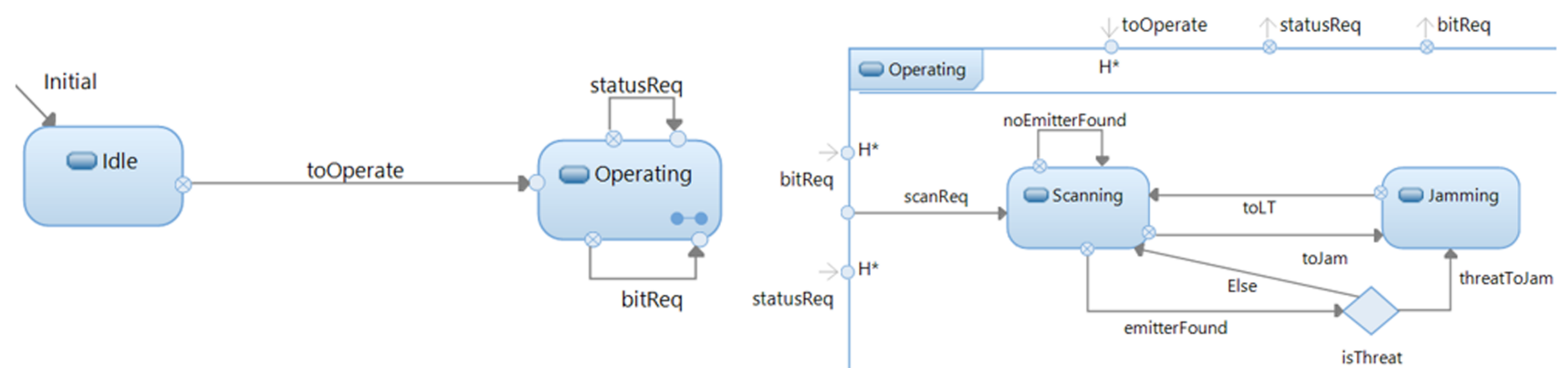

Fig. 9: Replacement of some self-transitions using extra model elements

Let us consider the design of a Controller capsule of an Electronic Warfare System in Figure 8. This capsule is responsible for coordinating message communication between two subsystems: Receiver and Jammer. When the scan timer fires, the Controller capsule sends a scan request to the Receiver capsule from the scanReq self-transition asking it to search for radar emitters in an electromagnetic spectrum. If an emitter is found, the Receiver sends it back to the Controller with the emitterFound signal and the Controller decides if it is a threat or a friendly emitter. If a threat is found, the Controller capsule sends it to the Jammer for jamming it. There are two modes of operation available in a Jammer: jamming and lookthrough $(L T)$. While the Jammer is in jamming mode, the Receiver must have to be in blanking mode, otherwise it can be damaged by the Jammer. On the other hand, the Receiver can be in the scanning mode only if the Jammer is in $L T$ mode. These requirements are implemented in this antipattern solution using two self-transitions toJam and toLT. In addition to these signals, the Controller also uses self-transitions for requesting subsystems for status information and built in test (BIT) in regular intervals.

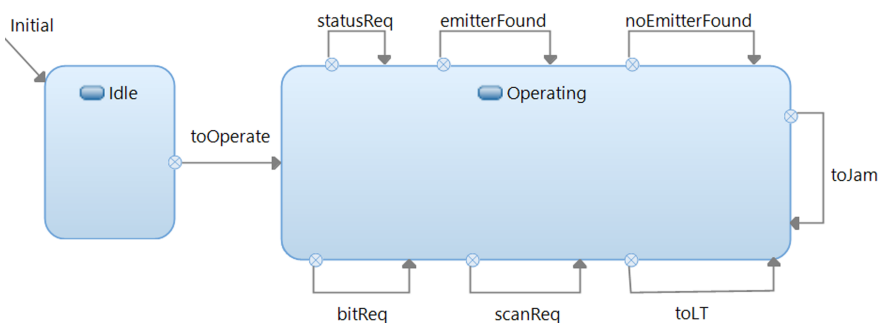

Fig. 8: Excessive use of self-transitions

Since all the communications in the Operating state are implemented using self-transitions, it is difficult to see the order of message handling in this behavioral diagram. For example, from this design it is not possible to predict whether the emitterFound signal can arrive at the Controller capsule before sending the scanReq signal.

\section{Symptoms and Consequences}

- Excessive use of self-transitions in the behavioral design of a model compared to the use of normal transitions.

- As the order of signal interaction is not clear, this antipattern directly affects understandability and maintainability of the design model.

\section{Refactored Solution}

This antipattern can be refactored by enforcing some order in the behavioral design. For example, Figure 9 demonstrates a design of the Controller capsule where it is easy to follow the order of execution of the message signals. This design improves the understandability of the model by introducing a set of new model elements inside the Operating state. For example, in contrast to the antipattern solution, this design gives us the ability to predict that the emitterFound signal can only be received in the Controller capsule after sending the scanReq signal.

\section{Known Exceptions}

There are situations when we might want to stay in the same state after handling a signal because of the design requirements. In these cases, the use of self-transitions is very handy. For example, in Figure 9, the use of self-transitions to request the subsystems for status information and BIT allows us to stay in the same state after responding to the events. In addition, self-transitions are also helpful in handling any unexpected signals [16].

\section{E. Misuse of Local Transitions \\ Background}

The origin of a local transition is the internal border of a state in a behavioral diagram. Local transitions can be used as self-transitions as well and therefore they are drawn using solid lines for distinguishing them from internal transitions. During the execution of a local transition, the exit and entry code of the source state do not execute. For example, in Figure 10. if the active state is a sub-state of the Operating state during the execution of the toOperate transition, the execution order would be as follows:

1) Exit code of the sub-state of the Operating state

2) Exit code of the Operating state

3) Effect of the toOperate transition

4) Entry code of the Operating state

5) Entry code of the sub-state of the Operating state

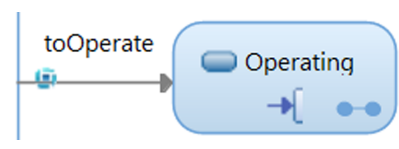

Fig. 10: Example of a local transition

\section{General Form}

Let us consider the design of a traffic-controller which can have three different types of traffic light: Red, Green, 
and Yellow. According to the requirement specifications, the activation of these traffic lights should follow this circular order: Green, Yellow, Red, Green ...

In Figure 11, a design choice for the top-level behavior of the traffic-controller capsule is shown. In this design choice, all the transitions are implemented as local transitions. Consequently, it is not clear if it is possible to go to the Red state directly from the Green state.

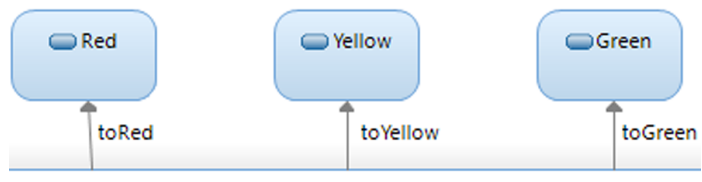

Fig. 11: Abuse of local transitions

\section{Symptoms and Consequences}

- Dominance of local transitions over other types of transitions.

- Since it is not possible to predict the sequence of signal interactions, this antipattern solution decreases understandability and maintainability.

\section{Refactored Solution}

The antipattern solution can be improved by replacing the local transitions with external transitions. As it is shown in Figure 12, the order of signal communication among different states is explicit due to the use of external transitions. The ease of following the order of communication makes this design choice much more understandable and one can easily rule out the possibility of going to the Red state directly from the Green state.

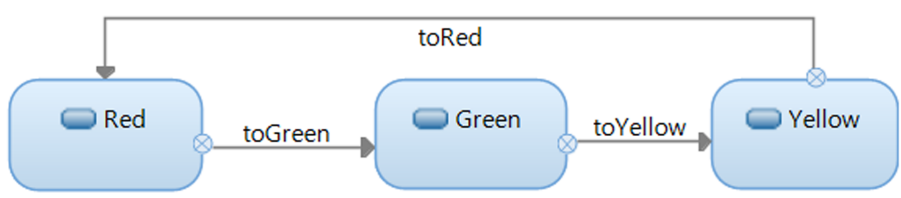

Fig. 12: Replacement of local transitions using external transitions

\section{Known Exceptions}

There are certain scenarios when we can have a large number of states in a single state diagram level and for following the requirement specification we need to go to a particular state regardless of our current active state after execution of an event trigger. In these scenarios, for simplifying our design model we can draw a local transition from the internal border of the enclosing state to the sub-state where we want to go. Therefore, in this case, the use of a local transition can save us from drawing transitions to the target state from all other states in the corresponding state diagram level.

\section{F. Incorrect use of asynchronous communication}

\section{Background}

There are two forms of communication available while designing a real-time software system: synchronous and asynchronous communication. There exists a tradeoff between

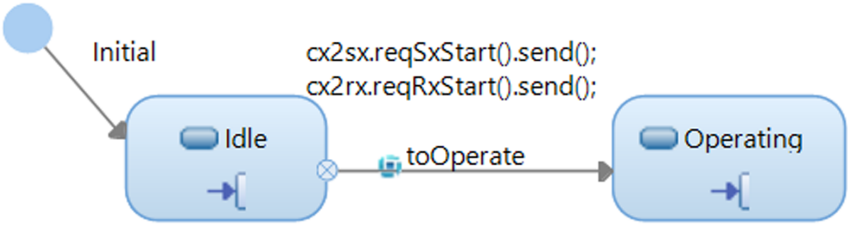

Fig. 13: Inappropriate use of asynchronous communication

concurrency and complexity while choosing a particular form of communication.

\section{General Form}

Let us consider the design of the top-level behavior of a Controller capsule in Figure 13 . Suppose, according to the design requirement, two subsystem capsules Sender and Receiver need to start executing before the Operating state becomes active. Due to the use of asynchronous communication in this antipattern, the delivery of the start signals to the subsystems is not guaranteed and no mechanism is implemented here for the Controller capsule to receive acknowledgement signals from the subsystems. Therefore, there exists a possibility that the Controller capsule would move to the Operating state without having started the subsystem capsules and thus the design requirement would be violated.

\section{Symptoms and Consequences}

- Since no assumptions can be made in UML-RT about when an asynchronous message is delivered, their use can lead to unexpected race conditions - such as bugs.

\section{Refactored Solution}

One way to avoid the dangers of asynchrony is to use synchronous communication. In Figure 13, if we replace the asynchronous send() with synchronous invoke() signals, the execution will be blocked in this transition until the Controller hears back from both of the Sender and the Receiver capsules.

\section{Consequences of the synchronous solution}

- It will require a fewer number of model elements for implementing the signal acknowledgement mechanism. Therefore, this refactored solution keeps the design simple compared to the asynchronous solution for this antipattern described below.

- The execution of all other capsules running in the same physical thread will be blocked until any reply is received by the sender of the synchronous signal. Therefore, the use of synchronous signals creates the possibility for the model to deadlock. One way to reduce this possibility is to send the signals again after waiting for a certain period of time, if no response has arrived.

\section{Variations}

If it is important that the sender of an asynchronous message is informed of successful delivery, acknowledgement messages can be used. Let us consider the example of asynchronous communication in Figure 14. In this example, a composite state is created in between the Idle and the 

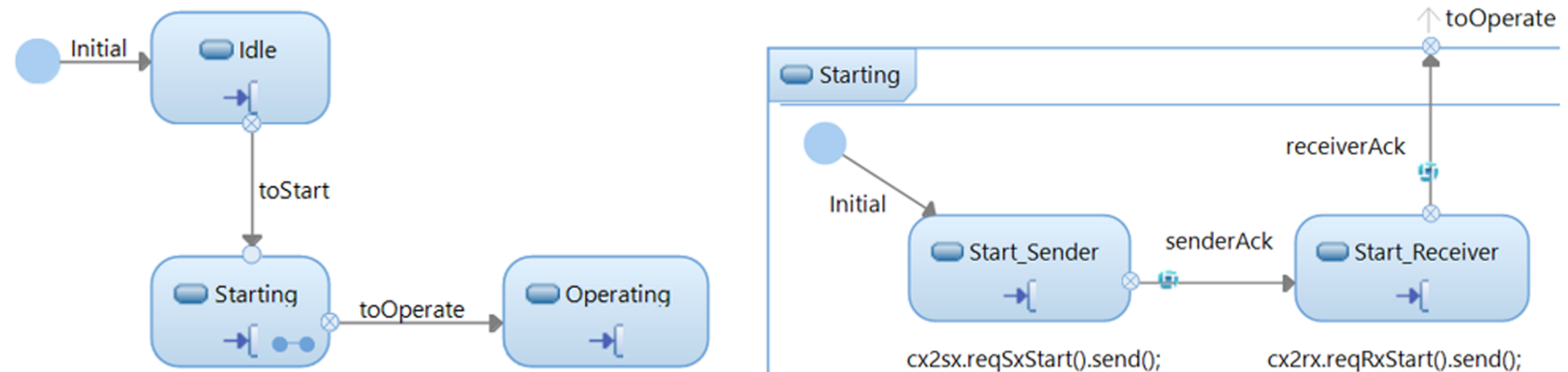

Fig. 14: Implementation of signal acknowledgement through asynchronous communication
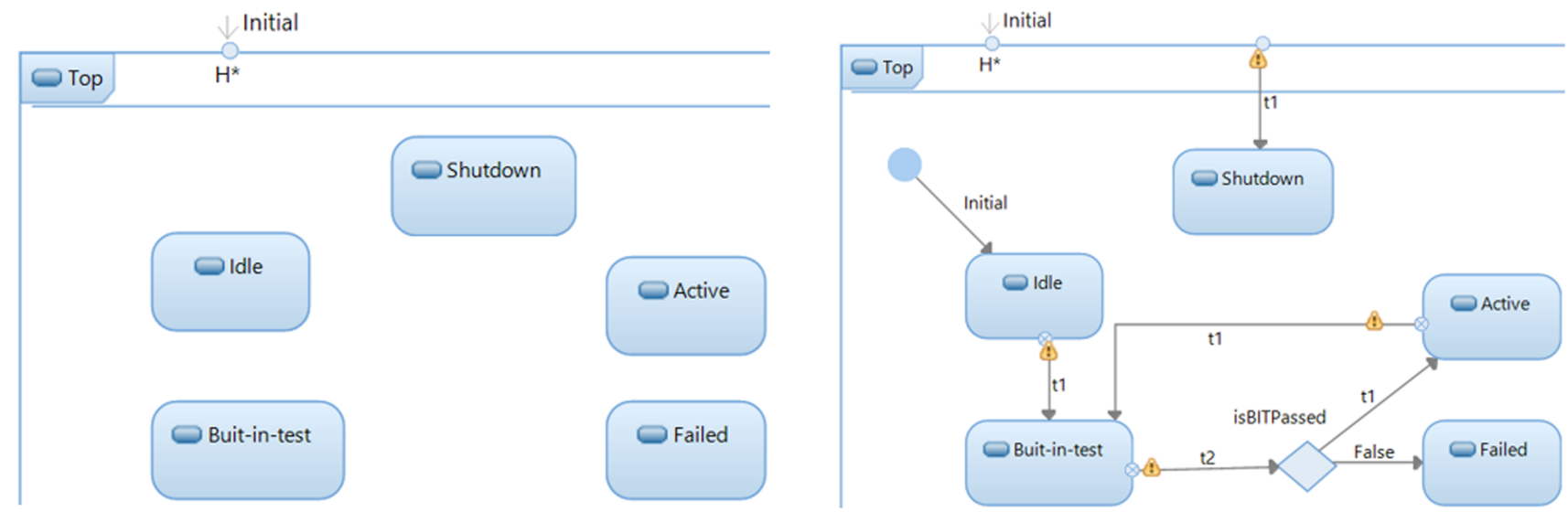

Fig. 15: Base-capsule behavior

Operating state which ensures that the subsystem capsules have started before the Controller reaches the Operating state.

Inside the Starting state, at first a start request is sent to the Sender from the Start_Sender state asynchronously. The execution will wait in this state until it receives an acknowledgement signal from the Sender capsule. Once it receives the acknowledgement, it will move to the Start_Receiver state and will follow the same approach for starting the Receiver. After getting reply from the Receiver capsule, the execution will go to the Operating state.

\section{Consequences of the asynchronous solution}

- Since all other capsules in the same physical thread are not blocked because of using asynchronous communication, it does not increase the potential for deadlocks.

- A high number of extra model elements is required for implementing signal acknowledgement compared to the synchronous solution.

Considering two different solutions for solving this antipattern, an obvious question is which solution is preferable? The answer to this question entirely depends on the preference of the designer between concurrency and complexity. Asynchronous communication is the preferred form of communication if designers want to minimize concurrency in the model. In addition, due to the blocking characteristic of synchronous communication, if it is used in the behavior of a capsule, the real-time aspects and response time for all other capsules within the same physical thread might be highly affected. However, if a simple design solution with fewer model elements if preferred for incorporating signal acknowledgement, then synchronous communication may still be a good choice.

\section{G. Absence of Inheritance}

\section{Background}

In object-oriented software development, inheritance allows the derivation of structural and behavioral properties from existing elements. The main purpose of using inheritance is to reuse properties that are common among a set of model elements and to implement an appropriate abstraction mechanism for dealing with complexity by allowing details to be introduced gradually. It is important to use inheritance properly as it can improve the understandability and maintainability of the design of a software model significantly. In UML-RT, inheritance is available in three forms: Structural inheritance, behavioral inheritance and protocol inheritance [29].

\section{General Form}

Let us consider the design of a simple real-time system where a set of sub-capsules are inheriting the top level behavior from a base-capsule. In Figure 15, two different versions of behavioral design of the base capsule are shown. According to the requirement specifications, the top level behaviors of the sub-capsules are identical, i.e., the corresponding states, transitions, events that trigger the transitions are the same in the top level behavior of all the sub-capsules.

Now, given these specifications, in Figure 15, the design choice on the left is obviously a poor one as it allows the 

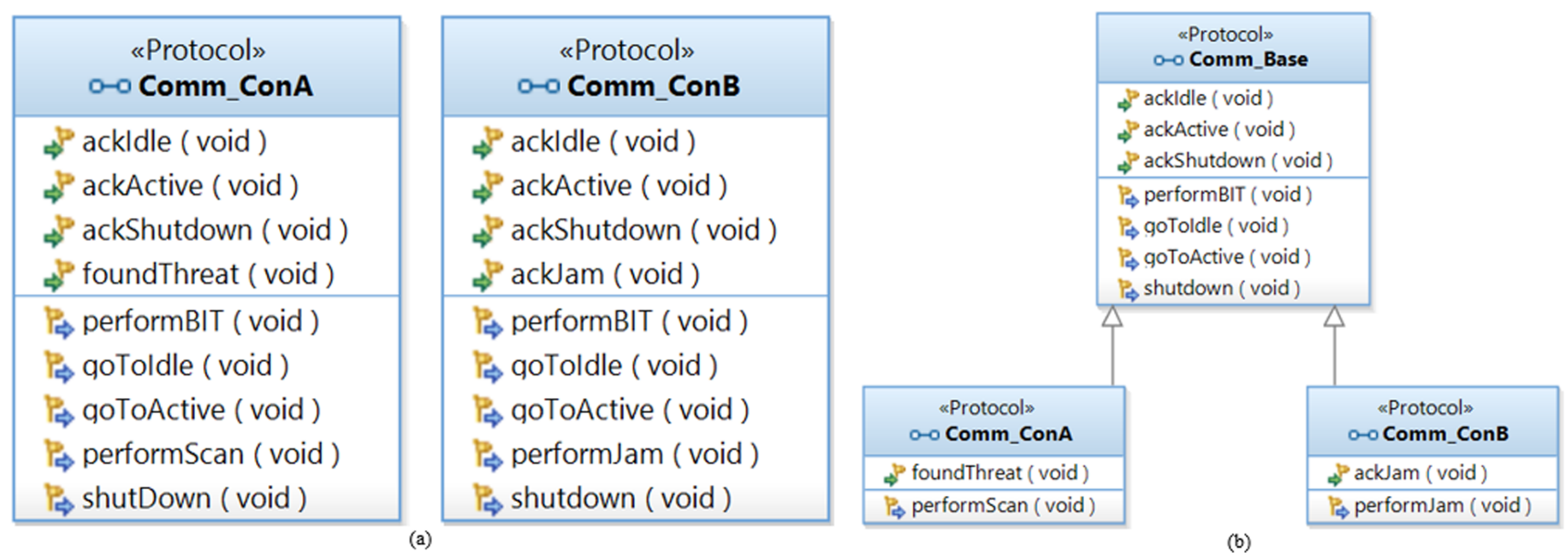

Fig. 16: (a) Absence of inheritance in protocol-design (b) Utilization of protocol inheritance

subsystems to inherit only some isolated states from the basecapsule. Consequently, the sub-capsules need to implement the remaining top level behavior by themselves, even though the behavior is similar in each of the sub-capsules. The design choice on the right is the better one compared to the design choice on the left. It allows the sub-capsules to inherit the transitions from the base-capsule. But, on the other hand, it does not allow the sub-capsules to inherit the trigger events which are also common among the sub-capsules.

Now, let us consider another example which illustrates the absence of inheritance in protocol design. Two protocols used in the design of a radar-jammer system are shown in Figure 16(a). As we can see, although these two protocols have three incoming signals and four outgoing signals in common, protocol inheritance is not utilized here.

\section{Symptoms and Consequences}

- Common model constructs among different capsules in the same abstraction level which are not inherited.

- Lack of inheritance affects readability and maintainability of the design model.

- This antipattern makes the model more error-prone as a future change in the design requirement involving common elements that have not been inherited requires the designers to make changes in each of the sub-capsules separately.

\section{Refactored Solution}

This antipattern can be improved by utilizing the inheritance feature more fully. As it is mentioned in [29], abstract classes in UML-RT are recommended to be complete and executable. It provides us the opportunity to obtain early feedback on its potential. In addition, an executable abstract representation can be used as a basis for discussing system requirements with customers and providing the development team members with a mean to have a high level understanding of the final implementation. Therefore, in this particular example, as the requirement specifications suggest identical top-level behavior for the sub-capsules, it would be considered as a good example of inheritance if all the common elements including states, transitions, trigger events and action code are inherited from the base capsule.

The refactored solution for the antipattern regarding protocol-inheritance is demonstrated in Figure 16(b). As we can see, a base protocol is created here which offers all the common incoming and outgoing signals to the derived protocols. Now the derived protocols only need to define the signals specific to their own implementation.

In addition to reusing the existing model elements, these refactored solutions enable us to make global changes in the derived constructs by changing the base modeling construct. This makes the design easier to understand and maintain.

\section{Known Exceptions}

There are certain scenarios in which it is preferable not to inherit all the possible model elements. For example, the design choice on the right of Figure 15 can be considered a better solution if it is possible that the requirements will change in the future in such a way that the sub-capsules need to use a different set of event triggers. In such cases, if we stick to this design choice, it would greatly improve the extensibility of the design model.

\section{CONCLUSION}

According to some statistics, nearly one-third of all software projects are cancelled, two-thirds of software projects suffer from cost overruns in excess of $200 \%$ and more than $80 \%$ of all software projects are considered failures [5]. One of the prominent reasons for the massive software failure rate is the poor software design quality. Since antipatterns are effective means to detect and refactor common software design mistakes, the understanding of antipatterns is important for maintaining good software quality. It is especially important in the development of real-time embedded software systems where the software complexity is extremely high. This paper introduces a set of seven antipatterns for the behavioral design of model-based real-time embedded software systems. For improving the understandability and enhancing the clarification of our discussion, the problematic design choice and the refactored solution for each of these antipatterns are described with examples. 
In the future, we will continue our work on identifying antipatterns, smells and patterns. Being motivated by the success of different code-oriented analyzers $[30]-[32]$, we are also planning to implement a tool which would allow developers to detect the presence of bad design choices automatically and refactor them accordingly.

\section{ACKNOWLEDGMENT}

We would like to thank Dr. Ron Smith from Royal Military College of Canada for giving us access to a student repository of UML-RT models. We would also like to thank our industrial research partner Ericsson Inc. for giving us access to some of their models. In addition, this work is supported in part by NSERC, as part of the NECSIS Automotive Partnership with General Motors, IBM Canada and Malina Software Corp.

\section{REFERENCES}

[1] R. France and B. Rumpe, "Model-driven development of complex software: A research roadmap," in (FOSE '07). IEEE Computer Society, 2007, pp. 37-54.

[2] B. Selic, G. Gullekson, J. McGee, and I. Engelberg, "ROOM: an objectoriented methodology for developing real-time systems," in ComputerAided Software Engineering, 1992. Proceedings., Fifth International Workshop, July 1992, pp. 230-240.

[3] B. Selic, "Using UML for modeling complex real-time systems," in (LCTES '98). Springer-Verlag, 1998, pp. 250-260.

[4] T. A. Henzinger and J. Sifakis, "The embedded systems design challenge," in (FM'06). Springer-Verlag Berlin, Heidelberg, 2006, pp. $1-15$.

[5] W. J. Brown, R. C. Malveau, H. W. McCormick, and T. J. Mowbray, AntiPatterns: Refactoring Software, Architectures, and Projects in Crisis. John Wiley \& Sons, Inc., 1998.

[6] Wikipedia, "Software quality," http://en.wikipedia.org/wiki/Software_ quality, [Online; accessed 30-April-2015].

[7] O. M. G. (OMG), "How to Deliver Resilient, Secure, Efficient, and Easily Changed IT Systems in Line with CISQ Recommendations," http: //www.omg.org/CISQ_compliant_IT_Systemsv.4-3.pdf, [Online; accessed 30-April-2015]

[8] I. O. for Standardization, "ISO/IEC 25000:2005, Software Engineering - Software product Quality Requirements and Evaluation (SQuaRE) - Guide to SQuaRE," http://www.iso.org/iso/catalogue_ detail.htm?csnumber=35683, [Online; accessed 05-April-2015].

[9] N. Leveson, "Medical Devices: The Therac-25," http://sunnyday.mit. edu/papers/therac.pdf, [Online; accessed 30-April-2015].

[10] WEBster, "Software Development Guidelines," http://www. literateprogramming.com/sdg.pdf, [Online; accessed 30-April-2015].

[11] Wikipedia, "Code smell," http://en.wikipedia.org/wiki/Code_smell, [Online; accessed 30-April-2015].

[12] Wikipedia, "Software design pattern," http://en.wikipedia.org/wiki/ Software_design_pattern, [Online; accessed 30-April-2015].

[13] Wikipedia, “Anti-pattern," http://en.wikipedia.org/wiki/Anti-pattern, [Online; accessed 30-April-2015].

[14] K. Project, "Software Development Guidelines," https://kepler-project. org/developers/reference/software-development-guidelines, [Online; accessed 30-April-2015]

[15] M. Fowler, Refactoring: Improving the Design of Existing Code. Addison-Wesley, 1999.

[16] M. Samek, Practical UML Statecharts in C/C++, Second Edition: Event-Driven Programming for Embedded Systems. Newnes Newton, MA, USA, 2008.

[17] B. P. Douglass, Doing hard time: developing real-time systems with UML, objects, frameworks, and patterns. Addison-Wesley Longman Publishing Co., Inc. Boston, MA, USA, 1999.

[18] M. Akroyd, "Antipatterns session notes." San Francisco, USA: Object World West, 1996.
[19] M. Abbes, F. Khomh, Y. Gueheneuc, and G. Antoniol, "An empirical study of the impact of two antipatterns, Blob and Spaghetti Code, on program comprehension,' in (CSMR '11). IEEE Computer Society, 2011, pp. 181-190.

[20] T. Parsons and J. Murphy, "Detecting performance antipatterns in component based enterprise systems," Journal of Object Technology, vol. 7, pp. 55-90, 2008 .

[21] F. Khomh, M. D. Penta, Y. Guéhéneuc, and G. Antoniol, "An exploratory study of the impact of antipatterns on class change- and faultproneness," Empirical Softw. Engg., vol. 17, no. 3, pp. 243-275, Jun. 2012.

[22] D. Romano, P. Raila, M. Pinzger, and F. Khomh, "Analyzing the impact of antipatterns on change-proneness using fine-grained source code changes," in (WCRE '12). IEEE Computer Society, 2012, pp. 437-446.

[23] S. E. S. Taba, F. Khomh, Y. Zou, A. E. Hassan, and M. Nagappan, "Predicting bugs using antipatterns," in (ICSM '13). IEEE Computer Society, 2013, pp. 270-279.

[24] C. U. Smith and L. G. Williams, "More new software performance antipatterns: Even more ways to shoot yourself in the foot," in $C M G$ Conference, 2011, pp. 717-725.

[25] V. Cortellessa, A. D. Marco, R. Eramo, A. Pierantonio, and C. Trubiani, "Digging into UML models to remove performance antipatterns," in (QUOVADIS '10). ACM, 2010, pp. 9-16.

[26] C. Trubiani and A. Koziolek, "Detection and solution of software performance antipatterns in palladio architectural models," in (ICPE 11). ACM, 2011, pp. 19-30.

[27] D. Arcelli, V. Cortellessa, and C. Trubiani, "Antipattern-based model refactoring for software performance improvement," in (QoSA '12). ACM, 2012, pp. 33-42.

[28] A. Neal, "RSARTE CheatSheet," https://www.ibm. com/developerworks/community/wikis/form/anonymous/ api/wiki/b7da455c-5c51-4706-91c9-dcca9923c303/page/ a7287c71-8e14-429d-80df-a0051a8b44b7/attachment/ 2eb38295-77c0-48e5-86e2-5903e4b4c224/media/cheatsheet._adam. pdf, [Online; accessed 30-April-2015].

[29] B. Selic, G. Gullekson, and P. T. Ward, Real-time Object-oriented Modeling. John Wiley \& Sons, Inc., 1994.

[30] GrammaTech, "FDA Recommends Static Analysis for Medical Devices," http://citeseerx.ist.psu.edu/viewdoc/download?doi=10.1.1. 194.293\&rep=rep1\&type=pdf, 2010, [Online; accessed 30-April-2015].

[31] N. Tsantalis, T. Chaikalis, and A. Chatzigeorgiou, "Jdeodorant: Identification and removal of type-checking bad smells," in (CSMR '08). IEEE Computer Society, 2008, pp. 329-331.

[32] R. P. Jetley, P. L. Jones, and P. Anderson, "Static analysis of medical device software using codesonar," in (SAW '08). ACM, 2008, pp. 22-29. 